\title{
Dielectric and polarization studies on some organic materials
}

\author{
B JAGANNADH and LALITHA SIRDESHMUKH \\ Physics Department, Kakatiya University, Warangal 506 009, India \\ MS received 1 December 1980

\begin{abstract}
Dielectric constant and dielectric loss $(\tan \delta)$ of oxamide, thiooxamide, urea, thiourea and some methyl and ethyl derivatives of urea and thiourea have been measured. The deformation polarization for all the materials is calculated from the static dielectric constants. The electronic contribution to molecular polarization has been evaluated theoretically using atomic hybridization components. The atomic polarization is obtained as the difference between the deformation polarization and electronic contribution to the deformation polarization.
\end{abstract}

Keywords. Dielectric constant; dielectric loss; molecular polarization.

\section{Introduction}

The data available on the dielectric properties of organic substances are meagre. The study of dielectric properties helps in understanding the nature of electric polarization. We report here the measurement of dielectric properties and molecular polarization in some organic solids. The deformation polarization has been calculated using the values of static dielectric constants. The electronic contribution to molecular polarization is evaluated semiemperically. The atomic contribution to the molecular polarization is obtained as the difference between the deformation polarization and electronic molecular polarization.

The substances studied are oxamide, thiooxamide and some alkyl ureas and thioureas.

\section{Experimental}

Urea obtained from BDH, thiourea from Sarabhai Merck Ltd. and substituted ureas supplied by Aldrich Chemical Co. Inc were recrystallized from methanol; oxamide and dithiooxamide obtained trom BDH were used without further purification. The dried samples were made into die-pressed pellets of about 3-4 mm thickness and $2.85 \mathrm{~cm}$ diameter. The dielectric constant and loss were measured at room temperature in the frequency range $50 \mathrm{~Hz}$ to $50 \mathrm{MHz}$. A Marconi Jig $155 / \mathrm{CI}$ was used in conjunction with a General Radio Electric Capacitance measuring assembly $1620 \mathrm{~A}$ in the frequency range $50 \mathrm{~Hz}$ to $50 \mathrm{kHz}$ and with a Marconi circuit magnification meter TF1245 in the frequency range $50 \mathrm{kHz}$ to $50 \mathrm{MHz}$. As the samples were highly hygroscopic, precautions were taken to avoid the effects of moisture. 
Many formulae have been proposed for the evaluation of the dielectric constant of a single crystal $\epsilon$ from the apparent dielectric constant $\epsilon_{p}$ measured on the powder samples. We have used the following relation suggested by Bottcher (1952)

$$
\epsilon=\frac{3 \delta \epsilon_{p}+2 \epsilon_{p}\left(\epsilon_{p}-1\right)}{3 \delta \epsilon_{p}-\left(\epsilon_{p}-1\right)}
$$

where $\delta$ is the packing fraction. Being soft, the organic substances get compressed well to form pellets of density nearly equal to the ideal density. The packing fraction for all the samples was of the order of 0.95 . Since the density values were not available for symm-dimethyl thiourea and symm-diethyl thiourea no porosity correction was applied. Plots of $\epsilon$ and $\tan \delta$ versus frequency are shown in figures (1-3). The dielectric constant decreases with increasing frequency beyond $10^{5} \mathrm{~Hz}$ it is frequency independent. The variations observed at low frequencies are due to factors like impurities, grain boundaries, defects, etc. In the case of oxamide and dithiooxamide, the dielectric constant is nearly frequency independent throughout the range covered in this investigation. This behaviour indicates that only electronic and atomic contributions for polarization are present (Rao and Smakula 1966). The frequency independent nature of $\epsilon$ for these two substances indicates that the samples are of very high purity. It can be seen that $\tan \delta$ decreases with frequency for all the substances studied and the value of loss at $10^{7} \mathrm{~Hz}$ is of the order of $10^{-2}$ for ureas and thioureas and $10^{-3}$ for oxamide and dithioxamide. Choudhury and Rao (1969) observed that

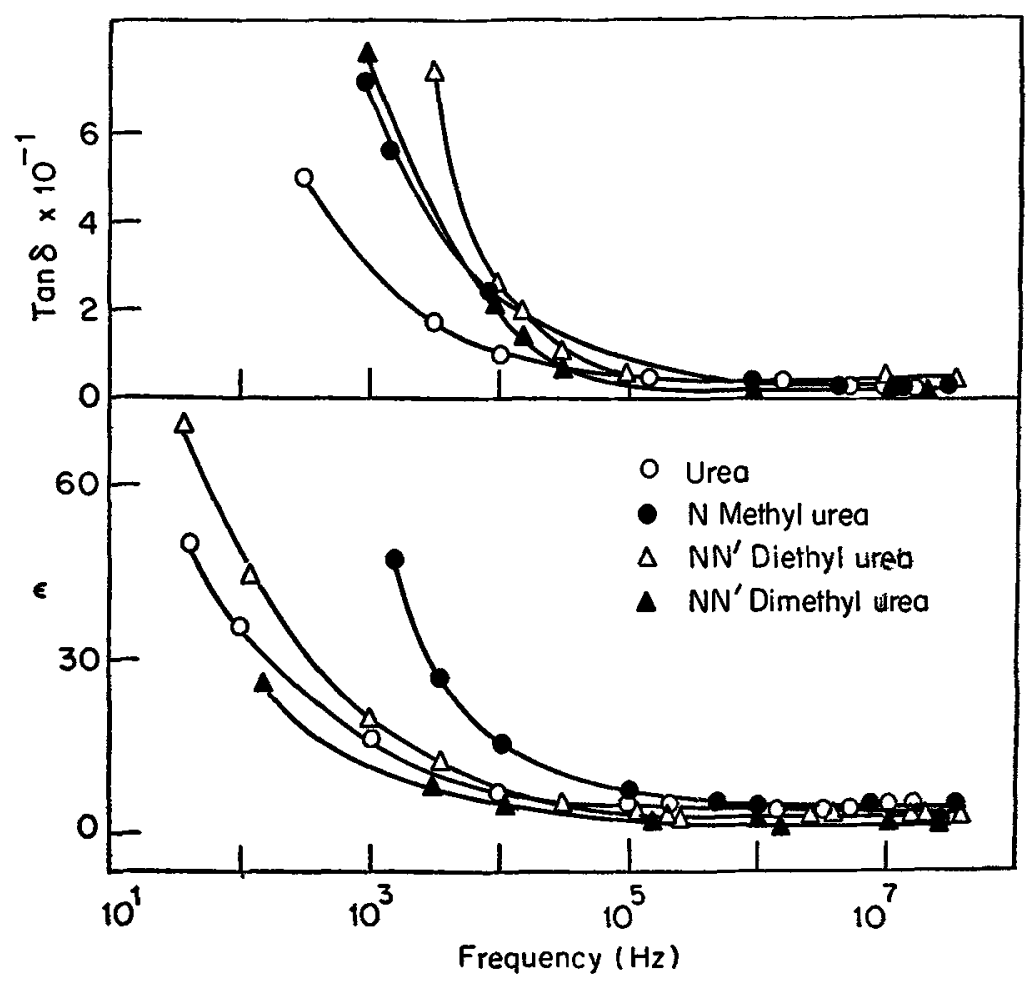

Figure 1. Variation of $\epsilon$ and $\tan \delta$ with frequency. 


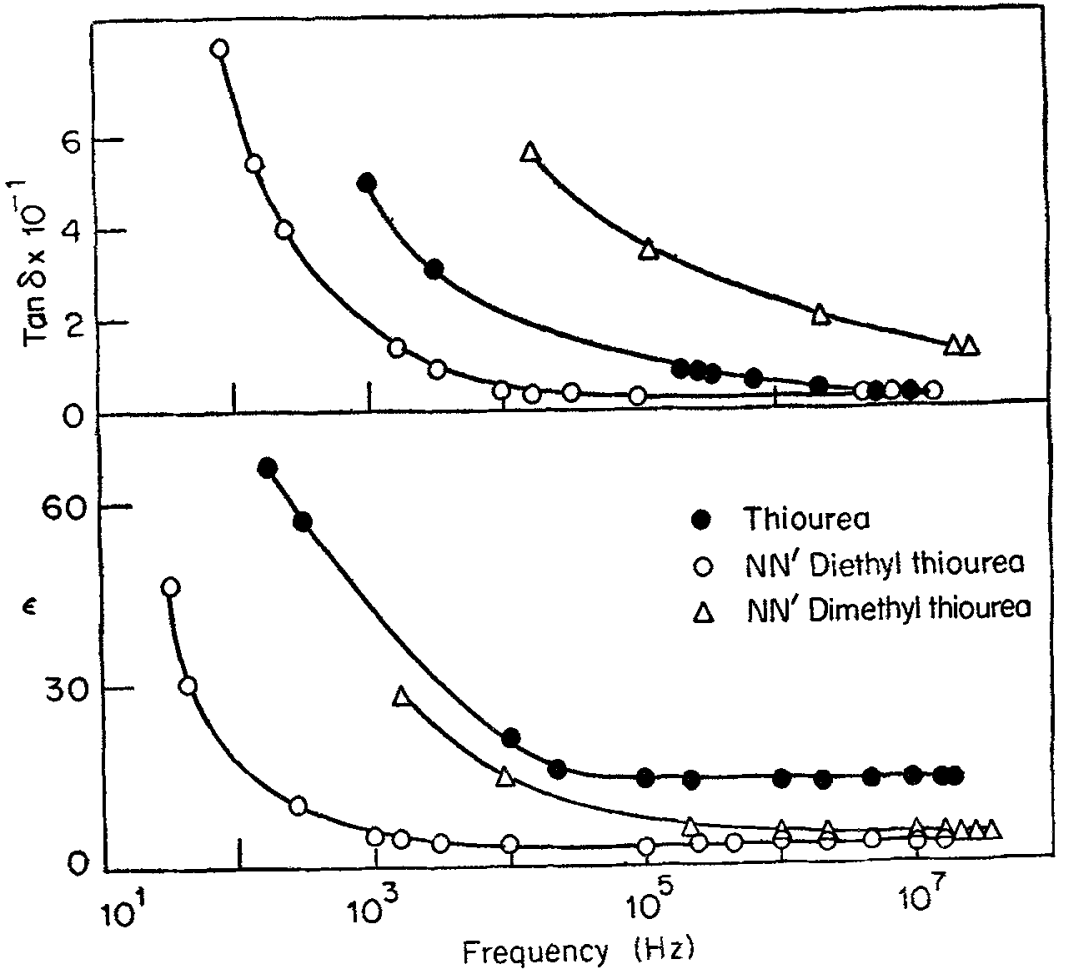

Figure 2. Variation of $\epsilon$ and $\tan \delta$ with frequency.

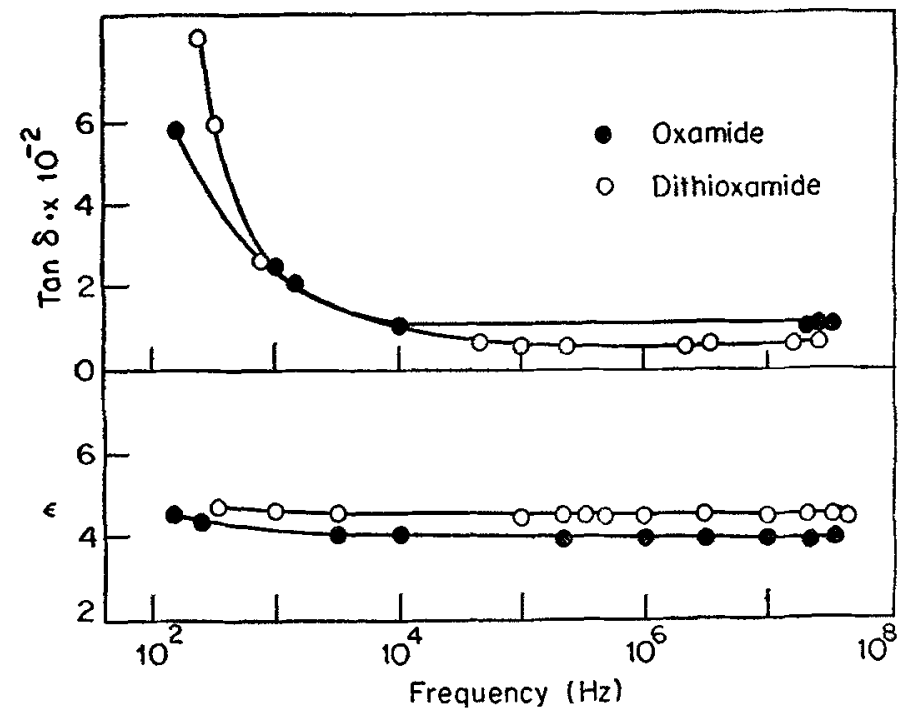

Figure 3. Variation of $\epsilon$ and $\tan \delta$ with frequency. 
the dielectric loss of the order of $10^{-2}$ indicates low concentration of defects. The frequency independent value is taken as the static dielectric constant. The values for all the substances are shown in table 1 using these values, the deformation polarization $P_{d}$ is calculated from the following relation (Smyth, 1955)

$$
P_{d}=\frac{\epsilon-1}{\epsilon+2} \frac{M}{d}
$$

where $M$ is the molecular weight, $d$ is the crystal density, $\epsilon$ is the average dielectric constant for which we have used the static dielectric constant. The values of $\boldsymbol{P}_{d}$ are shown in table 1.

\subsection{Molecular polarization}

Molecular polarization obtained from Clausius-Mosotti relation (equation 1) constitutes the sum of three contributions. They are the electronic, atomic and orientation polarization. In the present case, since the measurements have been done on solids the contribution due to the orientation polarization does not arise. The deformation polarization is the sum of electronic and atomic polarization $P_{e}$ and $P_{a}$ respectively. Further the molecular polarization can be expressed in terms of molecular polarizabilities; $a_{e}$ and $a_{a} \cdot a_{e}$ the electronic molecular polarizability and $a_{a}$ the atomic molecular polarizability. Thus

$$
P_{d}=P_{e}+P_{a}=4 / 3 \pi \mathrm{N}\left(a_{e}+a_{a}\right)
$$

The electronic molecular polarization is generally obtained from the LorentzLorentz equation using the data on refractive index (Smyth 1955). As experimental values of refractive index are not available for all the substances under study, we have used a semiempirical method proposed recently by Miller and Savchik (1979) to evaluate $P_{e}$.

\begin{tabular}{|c|c|c|c|c|c|}
\hline Substance & $\epsilon$ & $d$ & $\boldsymbol{P}_{d}$ & $P_{e}$ & $\boldsymbol{P}_{\boldsymbol{a}}$ \\
\hline Urea & $4 \cdot 09$ & $1 \cdot 323^{a}$ & $23 \cdot 03$ & $12 \cdot 68$ & $10 \cdot 36$ \\
\hline N methyl urea & $4 \cdot 18$ & $1 \cdot 204^{b}$ & $31 \cdot 69$ & $17 \cdot 22$ & $14 \cdot 47$ \\
\hline $\mathrm{NN}^{\prime}$ dimethyl urea & $3 \cdot 47$ & $1 \cdot 142^{a}$ & $34 \cdot 84$ & $21 \cdot 8$ & $13 \cdot 04$ \\
\hline $\mathrm{NN}^{\prime}$ diethyl urea & 2.89 & $1 \cdot 0415^{a}$ & $43 \cdot 39$ & $31 \cdot 03$ & $12 \cdot 36$ \\
\hline Thiourea & $10 \cdot 20$ & $1 \cdot 405^{a}$ & 41.08 & 20.85 & $20 \cdot 23$ \\
\hline $\mathrm{NN}^{\prime}$ dimethyl thiourea & $4 \cdot 04$ & $1 \cdot 074 *$ & $46 \cdot 38$ & $30 \cdot 15$ & $16 \cdot 23$ \\
\hline $\mathrm{NN}^{\prime}$ diethyl thiourea & $3 \cdot 2$ & $1.077^{*}$ & $49 \cdot 43$ & $39 \cdot 40$ & 10.03 \\
\hline Oxamide & 3.9 & $1 \cdot 667 c$ & 25.97 & $17 \cdot 71$ & $8 \cdot 26$ \\
\hline dithiooxamide & $4 \cdot 49$ & $1 \cdot 66^{d}$ & 46.03 & $34 \cdot 15$ & $11 \cdot 88$ \\
\hline
\end{tabular}

Table 1. The values of dielectric constant, density and polarization.

a Gregg E C 1973; b Mullen D and Hellner E 1978; c Ayerst E M and Duke J R C 1954;

d Wheatley P J 1965.

* Estimated from the measured density, assuming the packing fraction to be 0.95 . 
The functional form of the empirical formula, based on the variational perturbation approach proposed by Hylleras (1930) and Hasse (1930) and approximated by Hirshfelder et al (1954) is as follows

$$
a_{e}=\alpha(\mathrm{ahc})=\frac{4}{N}\left[\Sigma_{A} \tau_{A}\right]^{2}(\AA)^{3}
$$

where $N$ is the number of electrons in the molecule and ahc refers to atomic hybrid components $\tau_{A}$ of $a$ for each atom in a particular hybrid configuration. The summation proceeds over all the atoms $A$ in the molecule. The optimum values of $\tau_{A}$ were obtained by them from a systematic analysis of common organic compounds. The parameter $\tau_{A}$ does not depend on atoms which are bonded to $A$ but depends on the type of bonding through the hybridization of atomic orbitals. The parameters used are from Miller and Savchik (1979). The values of $P_{e}$ are shown in table 1. The values of the atomic contribution $P_{a}$ are also shown in table 1 .

\section{Discussion}

The electronic contribution to molecular polarization $P_{e}$ may be evaluated by using the semiempirical delta function developed by Lippincott and Daynhoft (1960) and employed by Lippincott and Stutman (1964), Nagarajan (1966), Lippincott et al (1968), Sanyal et al (1973), Murthy et al (1979). It was observed by Miller and Savchik (1979) that for a given system containing $M$ atoms the method of bond polarizabilities require $M(M+1) / 2$ parameters in contrast to the ahc method which requires less than $4 M$ parameters. As such it is found more convenient to use for large molecules. The ahc method could also give results in agreement with the experimental values. They observed that the errors resulting from the use of ahc method for a series of amides are smaller than those obtained by the Lippincott delta function method. In view of this we have used ahc method for evaluating $P_{e}$.

The values of atomic polarization $P_{a}$ are higher when compared to the values generally observed for a non-polar molecule. For non-polar molecules $P_{a}$ is $10-15 \%$ of the electronic polarization whereas for some molecules with polar groups the values are as high as $60 \%$ of the electronic polarization (Hill et al 1969). The high values can be attributed to the bending motion of polar groups. The molecules with balanced dipoles are found to have large $P_{a}$ values. The magnitude of $P_{a}$ depends on the size and mass of the atoms forming the dipoles. This gives rise to a higher value when sulphur is substituted for oxygen. This is evident from the values shown in table 1 . An increase is observed in passing from urea to the corresponding thioanalogue. Similar change is observed from oxamide to thiooxamide. A reduction in atomic polarization on symn ethyl substitution is observed both for urea and thiourea. But a gradual increase is indicated for monomethyl and dimethyl substitutions both for urea and thiourea. Further investigations are required to understand the cause for such a variation.

Apart from the dielectric constant of urea (Gregg 1973) and thiourea which have been extensively studied (Goldsmith and White 1959), there is no information regarding the dielectric constant measurements on the substituted ureas and thioureas 
and likewise on oxamide and dithiooxamide. There is a large difference in the dielectric behaviour of urea and thiourea while the latter is ferroelectric with a transition temperature below $0^{\circ} \mathrm{C}$, the former is not so. From table 1 , we find that there is a large difference in dielectric constant of urea and thiourea. But the values for substituted ureas and thioureas are of the same order as that of urea. These have much lower values when compared to thiourea. The present measurements have been done at room temperature. A study at lower temperatures would be of interest with regard to any possible ferroelectric behaviour. Further studies in this direction are being undertaken.

\section{Acknowledgements}

The authors thank Prof. D B Sirdeshmukh for his interest and encouragement in the present work and to Miss Prameela for her assistance in taking measurements.

\section{References}

Ayerst E M and Duke J R C 1954 Acta Crystallogr. 7588

Bottcher C J F 1952 Theory of electric polarization (Amsterdam: Elsevier)

Choudhury A K and Rao K V 1969 Phys. Stat. Solidi 32731

Goldsmith G J and White J G 1959 J. Chem. Phys. 311175

Gregg E C 1973 Handbook of physics and chemistry (Cleveland: CRC press)

Hasse H R 1930 Proc. Cambridge Philos. Soc. 26542

Hill N E, Vaughan W E, Price A H and Davies M 1969 Dielectric properties and behaviour (London: Van Nostrand Reinhold)

Hirshfelder J O, Curtiss C F and Bird R B 1954 Molecular theory of gases and liquids (New York: Wiley)

Hylleras 1930 Z. Phys. 65209

Lippincott E R and Daynhoff M O 1960 Spectrochim. Acta 16807

Lippincott E R, Nagarajan G and Stutman J M 1968 J. Phys. Chem. 7078

Lippincott E R and Stutman J M 1964 J. Phys. Chem. 682926

Miller K J and Savchik J A 1979 J. Am. Chem. Soc. 1017206

Mullen D and Hellner E 1978 Acta Crystallogr. B34 2789

Murthy D V, Subbiah D V and Naidu S V 1979 Indian J. Biochem. Biophys. 1643

Nagarajan G 1966 Indian J. Pure Appl. Phys. 4244

Rao K V and Smakula A 1966 J. Appl. Phys. 37319

Sanyal N K, Ahmad P and Dixit L 1973 J. Phys. Chem. 772552

Smyth C P 1955 Dielectric behaviour and structure (New York: McGraw Hill)

Wheatley P J 1965 J. Chem. Soc. part I 396 